Pacific Journal of Mathematics

SEQUENCING AND STARTERS

Vol. 64 , No. 1

May 1976 


\title{
SEQUENCINGS AND STARTERS
}

\author{
B. A. ANDERSON
}

B. Gordon characterized sequenceable Abelian groups as those Abelian groups with a unique element of order 2. In this paper Gordon's argument is generalized to prove that there are non-Abelian sequenceable groups of arbitrarily large even order. It is also noted that the sequencings described by Gordon are related to 1-factorizations of complete graphs and to Howell Designs.

1. Introduction. Suppose $G$ is a finite group of order $n$ with identity $e$. A sequencing of $G$ is an ordering $e, a_{2}, \cdots, a_{n}$ of all the elements of $G$ such that the partial products $e, e a_{2}, e a_{2} a_{3}, \cdots, e a_{2} \cdots a_{n}$ are distinct and hence also all of $G$. Sequencings arose in connection with the problem of constructing complete Latin Squares [5]. Later [8] it was noticed that sequencings can be used to decompose complete directed graphs into directed Hamiltonian paths. Other possible uses of sequencings are described here. It turns out that the sequencings of Gordon all induce 1-factorizations of an appropriate complete graph via associated "starters" [6, p. 176-177]. Certain sequencings and their "starters" also induce Howell Designs of type $H(2 m-2,2 m)$ by the "starter-adder" method [6, 176-177]. Thus, it appears that sequencings might have a broader applicability that has yet been recognized.

As mentioned above, sequenceable Abelian groups have been characterized [5]. But the sequencing question for non-Abelian groups has hardly been budged. Keedwell [7] reports that there are 9 known sequenceable non-Abelian groups and apparently in 7 of these cases, the results were determined by computer. Recently [2] other non-Abelian groups have been shown sequenceable. In this paper known sequencings and Gordon's original argument are used to construct infinite families of sequenceable non-Abelian groups of even order.

The sequencings we construct have the following property.

Definition 1. Suppose $G$ is a group of order $2 n$ with identity $e$ and unique element $g^{*}$ of order 2 . A sequencing $e, a_{2}, \cdots, a_{n}, \cdots, a_{2 n}$ will be called a symmetric sequencing iff $a_{n+1}=g^{*}$ and for $1 \leqq i \leqq n-1$, $a_{n+1+i}=\left(a_{n+1-i}\right)^{-1}$.

If $g^{*}$ is the unique element of order 2 in $G$, then $g^{*}$ is in the center of $G$. Thus, symmetric sequencings

$$
S: e, a_{2}, \cdots, a_{n}, a_{n+1}, a_{n}^{-1}, \cdots, a_{2}^{-1}
$$


have the associated partial product sequence

$$
P: e, b_{2}, \cdots, b_{n}, b_{n} g^{*}, b_{n-1} g^{*}, \cdots, b_{2} g^{*}, g^{*} \text {. }
$$

Definition 2. Suppose $G$ is a group of order $2 n$ with identity $e$ and unique element $g^{*}$ of order 2 . Then $E=\left\{\left\{x_{1}, y_{1}\right\}, \cdots,\left\{x_{n-1}, y_{n-1}\right\}\right\}$ is a left (right) even starter for $G$ iff

(i) every nonidentity element of $G$ except one, denoted $m$, occurs as an element of some pair of $E$,

(ii) every nonidentity element of $G$ except $g^{*}$ occurs in

$$
\left\{x_{\imath}^{-1} y_{\imath}, y_{i}^{-1} x_{l}: 1 \leqq i \leqq n-1\right\}\left(\left\{x_{\imath} y_{i}^{-1}, y_{\imath} x_{\imath}^{-1}: 1 \leqq i \leqq n-1\right\}\right) .
$$

If $G$ is Abelian, there is no distinction between left and right even starters. Even starters are a modification of the starter concept that has proven so useful in the construction of Room Squares [9] and perfect 1-factorizations $[1,3]$. Preliminary computer testing by the author and D. Morse indicates that even starters are more likely to generate perfect 1-factorizations than are starters. Thus the study of even starters should be of interest. But whereas it is easy to see that one always has the so-called patterned starter for groups of odd order, it does not appear to be trivial to show that all finite groups with a unique element of order 2 have an even starter. It turns out that such a group has a symmetric sequencing if and only if it has an even starter with an additional property. Thus, we can think of even starters as a kind of generalized sequencing. In order to see these results, we need to outline the construction of Gordon [5].

Suppose $G$ is sequenceable Abelian of order $2 n$. Then $G=A \times B$ where $A$ is cyclic of order $2^{k}, k>0$ and $B$ has odd order. $G$ has a basis $c_{0}, c_{1}, \cdots, c_{m}$ where $c_{0}$ has order $2^{k}$ and the orders $\delta_{1}, \delta_{2}, \cdots, \delta_{m}$ of $c_{1}, c_{2}, \cdots, c_{m}$ are odd positive integers such that $0<i<m$ implies $\delta_{t} \mid \delta_{t+1}$. If $j$ is any positive integer, then there exist unique integers $j_{0}, j_{1}, \cdots, j_{m}$ such that

$$
\begin{aligned}
& j \equiv j_{0}\left(\bmod \delta_{1} \delta_{2} \cdots \delta_{m}\right) \quad \text { and } \\
& j_{0}=j_{1}+j_{2} \delta_{1}+j_{3} \delta_{1} \delta_{2}+\cdots+j_{m} \delta_{1} \cdots \delta_{m-1}, \quad \text { where } \\
& 0 \leqq j_{1}<\delta_{1}, \quad 0 \leqq j_{2}<\delta_{2}, \cdots, 0 \leqq j_{m}<\delta_{m} .
\end{aligned}
$$

The sequence of partial products $P$ is defined as follows.

$$
\text { If } i=2 j+1,0 \leqq j<n, \text { then } b_{2 j+1}=c_{0}^{-1} c_{1}^{-J_{1}} c_{2}^{-j_{2}} \cdots c_{m}^{-j_{m}} \text {. }
$$

$$
\text { If } i=2 j+2, \quad 0 \leqq j<n, \text { then } b_{2 \jmath+2}=c_{0}^{j+1} c_{1}^{j_{1}+1} c_{2}^{l_{2}+1} \cdots c_{m}^{J_{m}+1} \text {. }
$$


The sequencing $S$ is defined as follows.

$$
\begin{aligned}
& \text { If } i=2 j+2, \quad 0 \leqq j<n \text {, then } a_{i}=b_{i-1}^{-1} b_{i}=c_{0}^{2 j+1} c_{1}^{2 j_{1}+1} \cdots c_{m}^{2 j_{m}+1} \text {. } \\
& \text { (3) If } i=2 j+1, \quad 0 \leqq j<n \text { and } s=\min \left\{r: j_{r} \neq 0\right\} \text {, then } a_{i}=b_{i-1}^{-1} b_{i} \\
& =c_{0}^{-2 j} c_{s}^{-2 J_{s}} c_{s+1}^{-2 j_{s+1}-1} \cdots c_{m}^{-2 j_{m}-1} \text {. If } j_{0}=0, \quad a_{\imath}=c_{0}^{-2 j} \text {. }
\end{aligned}
$$

2. Even starters and symmetric sequencings. We first show that the sequencings of Gordon are symmetric.

THeORem 1. Suppose $G$ is a sequenceable Abelian group of order $2 n$. The sequencing $a_{1}, a_{2}, \cdots, a_{2 n}$ described in (3) is a symmetric sequencing of $G$.

Proof. Suppose $a_{i} a_{k}=e$. Since $c_{0}$ has order $2^{k}, k>0$, it is clear from (3) that either $i$ and $k$ are both even or $i$ and $k$ are both odd. Suppose $i=2 j+1$ and $k=2 l+1,0 \leqq j, l<n$. By Definition 1 , it will suffice to show that $i+k \in\{2,2 n+2\}$. Clearly $j_{0}=0$ iff $l_{0}=0$. We use the argument of Gordon on the case $j_{0} \neq 0 \neq l_{0}$. By (3)

$$
a_{t}=c_{0}^{-2 j} c_{s}^{-2 J_{s}} c_{s+1}^{-2 j_{s+1}-1} \cdots c_{m}^{-2 J_{m}-1}
$$

and

$$
a_{k}=c_{0}^{-2 l} c_{t}^{-2 l_{1}} c_{t+1}^{-2 l_{t+1}-1} \cdots c_{m}^{-2 l_{m}-1}
$$

Now each $\delta_{i}$ is odd so that we must have $s=t$. Hence

$$
2(j+l) \equiv 0\left(\bmod 2^{k}\right)
$$

and

\section{Thus}

$$
\begin{array}{rlr}
j_{s}+l_{s} & \equiv 0\left(\bmod \delta_{s}\right) & j_{s}+l_{s}=\delta_{s} \\
j_{s+1}+l_{s+1}+1 & \equiv 0\left(\bmod \delta_{s+1}\right) & j_{s+1}+l_{s+1}+1=\delta_{s+1} \\
\vdots & \vdots \\
j_{m}+l_{m}+1 \equiv 0\left(\bmod \delta_{m}\right) . & j_{m}+l_{m}+1=\delta_{m} .
\end{array}
$$

If we multiply the $\delta_{s+i}$ equation by $\delta_{1} \cdots \delta_{s+t-1}$ and add, then by (1) we have $j_{0}+l_{0}=\delta_{1} \cdots \delta_{m}$. Thus $j+l \equiv 0\left(\bmod \delta_{1} \cdots \delta_{m}\right)$ and $2(j+l) \equiv$ $0(\bmod 2 n)$. The restrictions on $j$ and $l$ allow us to conclude that either $2(j+l)=0$ in which case $a_{i}=a_{k}=e$ or $2(j+l)=2 n$ which implies $j+l=n$ and $i+k=2 n+2$. The case where both $i$ and $k$ are even is similar.

We need some additional notation before the statement of the next 
theorem. If $E$ is an even starter for $G$, let $E^{*}=E \cup\{e, m\}$ and let $Q^{*}=\left\{\left\{x, x g^{*}\right\}: x \in G\right\}$. If we think of the elements of $G$ as labelling the complete graph $K_{|G|}$, then it is clear that $E^{*}$ and $Q^{*}$ are disjoint 1-factors of $K_{|G|}$ when $m \neq g^{*}$ and we may consider the 2-factor union of $E^{*}$ and $Q^{*}$.

THEOREM 2. The group $G$ has a symmetric sequencing iff $G$ has a left even starter $E$ such that $E^{*} \cup Q^{*}$ is a Hamiltonian circuit of $K_{|G|}$.

Proof. Suppose $|G|=2 n$ and $G$ has a symmetric sequencing with partial product sequence $b_{1}, b_{2}, \cdots, b_{2 n}$. Let

$$
\begin{aligned}
E= & \left\{\left\{b_{2 j+2}, b_{2(j+1)+1}\right\}: 0 \leqq j<\frac{n}{2}-1\right\} \\
& \cup\left\{\left\{b_{2 n-(2 j+1)}, b_{2 n-2 j}\right\}: 0 \leqq j \leqq \frac{n}{2}-1\right\} .
\end{aligned}
$$

Thus $m$ is $b_{n}$ if $n$ is even and $b_{n+1}$ otherwise. We must show that $E$ is a left even starter for $G$. For $0 \leqq j<n / 2-1,\left(b_{2 j+2}\right)^{-1} b_{2(j+1)+1}=a_{2(j+1)+1}=a_{p}$ where $3 \leqq p<n+1$ and $p$ odd. On the other hand $0 \leqq j \leqq n / 2-1$ implies $\left(b_{2 n-(2 \jmath+1)}\right)^{-1} b_{2 n-2 \jmath}=a_{2 n-2 j}=a_{q}$ where $n+2 \leqq q \leqq 2 n$ and $q$ is even. Since we have a symmetric sequencing it is clear that the $a_{p}$ 's and $a_{q}$ 's give us a set $C$ of $n-1$ distinct elements of $G \backslash\left\{e, g^{*}\right\}$. Furthermore, it is certainly impossible to get $2 n+2$ as the sum of two $p$ 's, two $q$ 's or a $p$ and a $q$. Thus no element of $C$ is the inverse of itself or another element of $C$ and $E$ is a left even starter for $G$. Note that since $g^{*}=b_{2 n}, g^{*} \neq m$. In each of the two cases $n$ even and $n$ odd it is easy to see that $E^{*} \cup Q^{*}$ is a Hamiltonian circuit of $K_{|G|}$.

Conversely suppose $G$ has a left even starter $E$ such that $E^{*} \cup Q^{*}$ is a Hamiltonian circuit of $K_{|G|}$. The circuit allows us to order the elements of $G$ by picking a starting vertex and direction around the circuit. Recall that $\{e, m\}$ is a pair of $E^{*}$. We start at $e$ and proceed around the circuit in the $m$ direction. For notational purposes we now use $h_{1}$ to denote $m$. Thus the Hamiltonian circuit is

$$
H: e, h_{1}, h_{1} g^{*}, h_{2}, h_{2} g^{*}, h_{3}, h_{3} g^{*}, \cdots, h_{n-1}, h_{n-1} g^{*}, h_{n}=g^{*} .
$$

Remember that $E^{*}=\left\{e, h_{1}\right\} \cup\left\{\left\{h_{\imath} g^{*}, h_{t+1}\right\}: 1 \leqq i \leqq n-1\right\}$. We use the sequence $H$ to construct the sequence $P$ of partial products. Again we have the two cases $n$ even and $n$ odd. In both cases we start $h_{1}=m$ in the middle $\left(a t b_{n}\right.$ or $\left.b_{n+1}\right)$ and work to the ends of $P$ alternating from side to side. For example, if $n$ is even, then

$$
P_{\text {even }}: e, h_{n-1}, h_{n-2} g^{*}, \cdots, h_{3}, h_{2} g^{*}, h_{1}, h_{1} g^{*}, h_{2}, h_{3} g^{*}, \cdots, h_{n-1} g^{*}, h_{n} \text {. }
$$


Note that $b_{n}=h_{1}=m$ and $b_{2 n}=h_{n}=g^{*}$. If $n$ is odd, then

$P_{\text {odd }}: e, h_{n-1}, h_{n-2} g^{*}, \cdots, h_{4}, h_{3} g^{*}, h_{2}, h_{1} g^{*}, h_{1}, h_{2} g^{*}, h_{3}, h_{4} g^{*}, \cdots, h_{n-1} g^{*}, h_{n}$.

Note that $b_{n+1}=h_{1}$ and $b_{2 n}=h_{n}$. In each case it is clear that $a_{n+1}=g^{*}$. Furthermore in each case it is clear that for $1 \leqq i \leqq n-1, a_{n+1-t}=$ $h_{i+1}^{-1} h_{i} g^{*}$ and $a_{n+1+i}=\left(a_{n+1-i}\right)^{-1}$. Finally $a_{n+1-i}$ is a difference associated with the starter pair $\left\{h_{i} g^{*}, h_{i+1}\right\}$ so that the sequence $a_{1}, \cdots, a_{2 n}$ includes every element of $G$ and is a symmetric sequencing.

THEOREM 3. If $G$ is a group with order $2 n$ and $E$ is a left even starter for $G$, then $E$ induces a 1-factorization $\mathscr{F}(E)$ on $K_{2 n+2}$.

Proof. Label the vertices of $K_{2 n+2}$ with the elements of $G$ and two ideal elements $\infty_{1}$ and $\infty_{2}$. Let $g^{*}, m$ and $e$ have their usual meanings. Note that here, $g^{*}$ and $m$ can be the same. Extend the group operation by defining for every $g$ in $G$ :

Suppose

$$
g \cdot \infty_{1}=\infty_{1} \cdot g=\infty_{1} \text { and } g \cdot \infty_{2}=\infty_{2} \cdot g=\infty_{2} .
$$

and

$$
E^{*}=E \cup\left\{e, \infty_{1}\right\} \cup\left\{m, \infty_{2}\right\}
$$

$$
Q^{*}=\left\{\left\{g, g^{*} g\right\}: g \in G\right\} \cup\left\{\infty_{1}, \infty_{2}\right\} \text {. }
$$

Let $\mathscr{F}(E)=\left\{g E^{*}: g \in G\right\} \cup Q^{*}$. It is obvious that each element of $\mathscr{F}(E)$ is a 1 -factor of $K_{2 n+2}$. Since $|\mathscr{F}(E)|=2 n+1$ it will suffice to show that every edge of $K_{2 n+2}$ occurs in some element of $\mathscr{F}(E)$. It is clear that all $\left\{g, \infty_{1}\right\}$ and $\left\{g, \infty_{2}\right\}$ belong to 1 -factors in $\mathscr{F}(E)$ and $\left\{\infty_{1}, \infty_{2}\right\} \in Q^{*}$. Thus, suppose $\{g, h\}$ is a pair of distinct elements of $G$. If $g^{-1} h=g^{*}$, then $h=g g^{*}=g^{*} g$ and $\{g, h\} \in Q^{*}$. If $g^{-1} h \neq g^{*}$ then there is a pair $\{x, y\} \in$ $E$ such that $g^{-1} h=x^{-1} y$. Thus $g x^{-1}=h y^{-1}$ so that if $k$ is this common element, $k \cdot\{x, y\}=\{g, h\} \in k E^{*}$ and the result follows.

A group $G$ may have even starters but have no even starter $E$ such that $E^{*} \cup Q^{*}$ is a Hamiltonian circuit. For example consider the quaternion group $Q_{3}$ with generators $a$ and $b$ and defining relations $a^{4}=e, \quad b^{2}=a^{2}$ and $b a=a^{3} b$. One may verify that $E=$ $\left\{\{a, a b\},\left\{a^{2} b, a^{3} b\right\},\left\{a^{3}, b\right\}\right\}$ is both a left and right even starter for $Q_{3}$. Note that here $g^{*}=a^{2}=m$ so that $E^{*} \cup Q^{*}$ is not a Hamiltonian circuit. It has been computer verified [4] that $Q_{3}$ has no sequencing whatsoever. Thus it certainly has no symmetric sequencing and no even starter $E$ such that $E^{*} \cup Q^{*}$ is a Hamiltonian circuit. Actually it is quite easy to prove algebraically that $Q_{3}$ does not have a symmetric sequencing. For, suppose $S$ : $e, a_{2}, a_{3}, a_{4}, g^{*}, a_{4}^{-1}, a_{3}^{-1}, a_{2}^{-1}$ is a symmetric sequencing of $Q_{3}$. Now $Q_{3} /\left\langle a^{2}\right\rangle \cong Z_{2} \times Z_{2}$. Let the members of $Z_{2} \times Z_{2}$ be 
designated by $e, 1,2,3$. Then each nonidentity element of $Z_{2} \times Z_{2}$ is its own inverse and the product of any two nonidentity elements is the third. The sequence $S$ must induce a sequence $T$ on $Z_{2} \times Z_{2}$ such that every element of $Z_{2} \times Z_{2}$ occurs exactly twice in $T$ and its associated partial product sequence $P$. Clearly $S$ forces $T$ to be of the form

$$
T: 1, x, y, z, 1, z, y, x \text {. }
$$

But then the associated partial product sequence is

$$
P: 1, x, z, 1,1, z, x, 1
$$

and $Q_{3}$ has no symmetric sequencing.

It seems possible that symmetric sequencings could be used to construct families of Howell Designs of type $H(2 m-2,2 m)$. For example consider the cyclic group $Z_{10}$. The symmetric sequencing

$$
S: 0,4,8,1,3,5,7,9,2,6
$$

induces the partial product sequence

$$
P: 0,4,2,3,6,1,8,7,9,5 \text {. }
$$

The associated even starter is $E=\{\{4,2\},\{3,6\},\{8,7\},\{9,5\}\}$. Note that the sums of the pairs of $E$ are $6,9,5,4$. The sums are distinct and no sum is $2 \cdot m=2 \cdot 1$. Thus, it follows from [6] that $-6,-9,-5,-4,-2,0$ when applied to the $\{4,2\},\{3,6\},\{8,7\},\{9,5\}, 1,0$ in order give a starter-adder construction of an $H(10,12)$. Thus, we arrive at the following.

Definition 3. Suppose $G$ is an Abelian group of order $2 n$ and $S$ is a symmetric sequencing of $G . S$ is strong if and only if in the associated partial product sequence $P$

and

(i) $1 \leqq i<j \leqq n-1$ implies $b_{\imath} b_{i+1} \neq b_{l} b_{l+1}$

(ii) $1 \leqq i \leqq n-1$ implies $e \neq b_{l} b_{\imath+1} \neq m^{2}$.

As noted above, a strong symmetric sequencing of an Abelian group of order $2 m-2$ will induce a Howell Design of type $H(2 m-2,2 m)$.

Problem. Find general constructions of strong symmetric sequencings.

Note that from (2) it follows easily that the sequencings of Gordon are not strong for $n \geqq 4$ since for those sequencings $0 \leqq j<n$ implies that $b_{2 j+1} \cdot b_{2 \jmath+2}=c_{0} c_{1} \cdots c_{m}$. 
We conclude the paper by generalizing the theorem of Gordon to include non-Abelian groups of arbitrarily large even order. Let $Z_{2}$ denote the group of order 2 .

THEOREM 4. If $G$ is a sequenceable group of odd order $n$, then $G \times Z_{2}$ has a symmetric sequencing.

Proof. By hypothesis, $G$ has a sequencing $e, x_{2}, \cdots, x_{n} . G \backslash\{e\}$ can be partitioned into 2-element subsets such that each subset consists of an element and its inverse. Choose one element from each 2-element subset. If $x_{i}$ is a chosen element, then associate $\left(x_{i}, 1\right)$ with $x_{i}$ and if $x_{i}$ is not a chosen element, associate $\left(x_{t}, 0\right)$ with $x_{i}$. This leads to the string

$$
y_{1}, y_{2}, \cdots, y_{n}=(e, 0),\left(x_{2}, i_{2}\right), \cdots,\left(x_{n}, i_{n}\right)
$$

of elements of $G \times Z_{2}$. We extend this to a symmetric sequencing of $G \times Z_{2}$ as follows. Define $y_{n+1}=g^{*}=(e, 1)$ and for $1 \leqq j \leqq n-1$, let $y_{n+1+j}=\left(y_{n+1-j}\right)^{-1}=\left(x_{n+1-j}^{-1}, i_{n+1-j}^{-1}\right)$. It is clear that the string $y_{1}, y_{2}, \cdots, y_{n}, \cdots, y_{2 n}$ includes all elements of $G \times Z_{2}$. Since $y_{n+1}=(e, 1)$ is certainly in the center of $G \times Z_{2}$, it is easy to see that the partial products also include all elements of $G \times Z_{2}$.

Note that by [7] there are at least 5 known sequenceable nonAbelian groups of odd order.

THEOREM 5. Suppose the group $G$ has a symmetric sequencing and $B$ is an Abelian group such that $\operatorname{gcd}(|G|,|B|)=1$. Then $G \times B$ has a symmetric sequencing.

Proof. The idea is to replace the cyclic group $A$ of order $2^{h}, h>0$ in [5] with $G$ and use the arguments of Gordon and Theorem 1. $G$ has order $2 n$. Let $S_{G}: x_{1}, x_{2}, \cdots, x_{2 n}$ be a symmetric sequencing of $G$ with associated partial product sequence $P_{G}: y_{1}, y_{2}, \cdots, y_{2 n}$. Now $B$ has odd order $k$. We wish to define a symmetric sequencing $S: a_{1}, a_{2}, \cdots, a_{2 n k}$ of $G \times B$. We first define the partial product sequence $P: b_{1}, b_{2}, \cdots, b_{2 n k}$. In the following we use the complete residue system $1,2,3, \cdots, 2 n \bmod 2 n$. As before $B$ has a basis $c_{1}, \cdots, c_{m}$ such that the orders $\delta_{1}, \cdots, \delta_{m}$ are odd positive integers with $0<i<m$ implying $\delta_{\imath} \mid \delta_{\imath+1}$ so that (1) still holds.

$$
\begin{aligned}
& \text { If } i=2 j+1, \quad 0 \leqq j<n k \text {, then } b_{2 \jmath+1}=\left(y_{t(\bmod 2 n)}, c_{1}^{-j_{1}} \cdots c_{m}^{-J_{m}}\right) \text {. } \\
& \text { If } i=2 j+2, \quad 0 \leqq j<n k \text {, then } b_{2 \jmath+2}=\left(y_{l(\bmod 2 n)}, c_{1}^{j_{1}+1} \cdots c_{m}^{j_{m}+1}\right) \text {. }
\end{aligned}
$$

The $b_{\imath}$ 's must be shown to be distinct. Suppose $b_{s}=b_{t}$ where $s=2 u+1$ and $t=2 v+1,0 \leqq u, v<n k$. Then clearly $2 u \equiv 2 v(\bmod 2 n)$ so that $u \equiv v(\bmod n)$. As in the proof of Theorem $1, u \equiv v(\bmod k)$. Since $\operatorname{gcd}(n, k)=1$, we have $u \equiv v(\bmod n k)$ and thus $u=v$. A similar argument suffices if $s=2 u+2$ and $t=2 v+2,0 \leqq u, v<n k$. Finally, if 
$s=2 u+1$ and $t=2 v+2$, then the first coordinates of $b_{s}$ and $b_{t}$ must be different.

The sequence $S$ is now defined as follows.

$$
\begin{aligned}
& \text { If } i=2 j+2,0 \leqq j<n k \text {, then } a_{t}=b_{i-1}^{-1} b_{l}=\left(x_{t(\bmod 2 n)}, c_{1}^{2 j_{1}+1} \cdots c_{m}^{2 j_{m}+1}\right) \text {. } \\
& \text { If } i=2 j+1,0 \leqq j<n k \text { and } s=\min \left\{r: j_{r} \neq 0\right\} \text {, then } a_{t}=b_{i-1}^{-1} b_{i} \\
& =\left(x_{t(\bmod 2 n)}, c_{s}^{-2 l_{s}} c_{s+1}^{-2 s_{s+1}-1} \cdots c_{m}^{-2 J_{m}-1}\right) \text {. If } j_{0}=0, \quad a_{t}=\left(x_{t(\bmod 2 n)}, e\right) \text {. }
\end{aligned}
$$

We may verify that the $a_{l}$ 's are distinct in the same way that the $b_{\imath}$ 's were shown distinct. Thus $G \times B$ has a sequencing.

The indicated sequencing is in fact symmetric. Suppose $a_{p} a_{q}=e$. The symmetric sequencing of $G$ shows that either $p$ and $q$ are both even or $p$ and $q$ are both odd. The argument now proceeds as in Theorem 1 .

It would be nice if the results above could be used to find sequencings of more non-Abelian groups of odd order. For example, it is known that the non-Abelian group $N_{21}$ of order 21 is sequenceable. Thus by Theorems 4 and $5, N_{21} \times Z_{2}$ and $\left(N_{21} \times Z_{2}\right) \times Z_{5}$ have symmetric sequencings. One might hope that the symmetric sequencings of $\left(N_{21} \times\right.$ $\left.Z_{2}\right) \times Z_{5}$ would "cut back" to a sequencing of $N_{21} \times Z_{5}$, but this is not the case in general.

Added in Proof. R. Friedlander in a paper to appear in Aequationes Math. has shown that if $p$ is a prime, $p \equiv 1(\bmod 4)$, then the dihedral group $D_{p}$ is sequenceable.

\section{REFERENCES}

1. B. A. Anderson, A Class of Starter-Induced 1-Factorizations, in Graphs and Combinatorics, Lecture Notes in Mathematics \#406, Springer-Verlag 1974, 180-185.

2. - Sequencings of Certain Dihedral Groups, Proc. of Sixth Southeastern Conf. on Comb., Graph Theory and Computing, Winnipeg, 1975, 65-76.

3. B. A. Anderson and D. Morse, Some Observations on Starters, Proc. of Fifth Southeastern Conf. on Comb., Graph Theory and Computing, Winnipeg, 1974, 229-235.

4. J. Dénes and E. Török, Groups and Graphs, in Combinatorial Theory and its Applications, North Holland, (1970), 257-289.

5. B. Gordon, Sequences in groups with distinct partial products, Pacific J. Math., 11 (1961), 1309-1313.

6. S. H. Y. Hung and N. S. Mendelsohn, On Howell designs, J. Combinatorial Theory (A), 16 (1974), 174-198.

7. A. D. Keedwell, Row complete squares and a problem of A. Kotzig concerning P-quasigroups and Eulerian circuits, J. Combinatorial Theory (A), 18 (1975), 291-304.

8. N. S. Mendelsohn, Hamiltonian decompositions of the complete directed n-graph, in Proc. Colloq. Tihany, Academic Press, (1968), 237-241.

9. W. D. Wallis, Room Squares, Lecture Notes in Mathematics \#292, Springer-Verlag, 1972.

Received July 30, 1975. This research was partially supported by an Arizona State University Summer Faculty Fellowship. 



\section{Pacific Journal of Mathematics}

Vol. 64, No. 1

May, 1976

Walter Allegretto, Nonoscillation theory of elliptic equations of order $2 n \ldots \ldots \quad 1$

Bruce Allem Anderson, Sequencings and starters.................. 17

Friedrich-Wilhelm Bauer, A shape theory with singular homology .......... 25

John Kelly Beem, Characterizing Finsler spaces which are

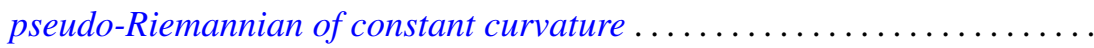

Dennis K. Burke and Ernest A. Michael, On certain point-countable

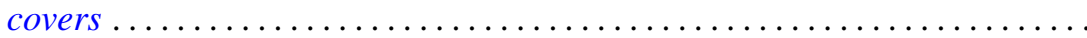

Robert Chen, A generalization of a theorem of Chacon ............... 93

Francis H. Clarke, On the inverse function theorem ................ 97

James Bryan Collier, The dual of a space with the Radon-Nikodým

property ....................................... 103

John E. Cruthirds, Infinite Galois theory for commutative rings ............ 107

Artatrana Dash, Joint essential spectra......................... 119

Robert M. DeVos, Subsequences and rearrangements of sequences in FK

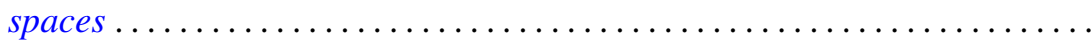

Geoffrey Fox and Pedro Morales, Non-Hausdorff multifunction generalization

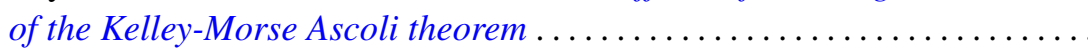

Richard Joseph Fleming, Jerome A. Goldstein and James E. Jamison, One

parameter groups of isometries on certain Banach spaces.............

Robert David Gulliver, II, Finiteness of the ramified set for branched

immersions of surfaces

Kenneth Hardy and István Juhász, Normality and the weak cb property ...... 167

C. A. Hayes, Derivation of the integrals of $L^{(q)}$-functions.

Frederic Timothy Howard, Roots of the Euler polynomials .

Robert Edward Jamison, II, Richard O'Brien and Peter Drummond Taylor, On

embedding a compact convex set into a locally convex topological vector space ....................................

Andrew Lelek, An example of a simple triod with surjective span smaller than span ...

Janet E. Mills, Certain congruences on orthodox semigroups

Donald J. Newman and A. R. Reddy, Rational approximation of $e^{-x}$ on the positive real axis.

John Robert Quine, Jr., Homotopies and intersection sequences ...

Nambury Sitarama Raju, Periodic Jacobi-Perron algorithms and fundamental units ....

Herbert Silverman, Convexity theorems for subclasses of univalent functions. . .

Charles Frederick Wells, Centralizers of transitive semigroup actions and endomorphisms of trees.........................

Volker Wrobel, Spectral approximation theorems in locally convex spaces ..................... 